\title{
Discriminating Between Surface and Bulk Recombination in Organic Solar Cells by Studying the Thickness Dependence of the Open-Circuit Voltage
}

\author{
Irene Zonno ${ }^{1,2}$, Benedikt Krogmeier ${ }^{1,2}$, Verena Katte ${ }^{2}$, Dana Lübke ${ }^{2}$, Alberto Martinez- \\ Otero $^{1,2}$, and Thomas Kirchartz ${ }^{1,2}$ \\ ${ }^{1}$ IEK5-Photovoltaik, Forschungszentrum Jülich, 52425 Jülich, Germany \\ ${ }^{2}$ Faculty of Engineering and CENIDE, University of Duisburg-Essen, Carl-Benz-Str. 199, 47057 Duisburg, \\ Germany
}

\begin{abstract}
In case of thin-film solar cells it is often rather difficult to determine what the dominant recombination mechanism is. In particular it is difficult to distinguish recombination at the interface between the absorber layer and the electrodes (typically called surface recombination) from recombination in the bulk of the absorber - or in case of organic solar cells at the internal donor-acceptor interfaces. Here, we suggest a method to distinguish surface and bulk recombination in thin-film solar cells based on the thickness dependence of the saturation current density, which we derive from the open-circuit voltage and the photocurrent at short circuit or reverse bias. By means of numerical simulations, we show that surface and bulk recombination currents scale differently with thickness assuming the material properties to be unchanged. We test our predictions on a range of organic solar cell data from our laboratory and from literature and show that in the field of organic photovoltaics the whole range of cases, from mostly surface limited to purely bulk limited, is observed.
\end{abstract}


Non-geminate recombination is of relevance for any solar cell technology because it is the main factor that determines the open-circuit voltage ${ }^{1-6}$. In many thin-film solar cells, nongeminate recombination might also substantially affect charge collection and therefore the solar cell fill factor and short circuit current ${ }^{7-11}$. Thus, identification of the main sources of the non-geminate recombination loss for a given device is of high general interest for the field of photovoltaics. In many cases, it may be possible to identify the dominant recombination mechanism, i.e. Shockley-Read-Hall, radiative or Auger, from an analysis of luminescence yield $^{12}$, luminescence transients ${ }^{13,14}$, ideality factor ${ }^{15-19}$, doping level ${ }^{17,20,21}$, and numerical simulations ${ }^{22,23}$. However, distinguishing between recombination at the interface between absorber and electrodes (surface recombination) and recombination in the bulk of the absorber (bulk recombination) can be extremely challenging ${ }^{24,25}$. In the case of crystalline silicon solar cells, surface recombination velocities can be derived from effective lifetimes measured on high quality floatzone wafers with extremely long bulk lifetimes ${ }^{26,27}$. This is possible essentially because one assumes that the surface is limiting the lifetime of the whole system and because the mobilities of crystalline $\mathrm{Si}$ are so well known that diffusion to the surface can be easily taken into account ${ }^{28}$. In case of thin-film solar cells, and especially when dealing with new material systems such as organic or perovskite based absorbers, mobilities, electrostatics, and bulk lifetimes might not be sufficiently well known to be able to take their influence into account quantitatively such that surface properties can be extracted from measurements of the effective lifetime of the whole device. Thus, there is a clear need to deal with the question of how to at least determine whether surface or bulk recombination is more dominant.

Here, we propose a method, based on the thickness dependence of the saturation current density, to distinguish between surface and bulk recombination in organic solar cells. By means of numerical simulations, we show that the total recombination current scales differently in a device dominated by surface or bulk recombination allowing one to assign the 
dominant recombination mechanism of the solar cells under investigation either to the surface or the bulk. We test this approach on a range of data from our laboratory and from literature showing that in the field of organic photovoltaics a broad range of cases, from mostly surface limited to purely bulk limited, can be observed.

Surface and bulk recombination currents depend differently on the absorber layer thickness. This is a relatively trivial observation that is based on the fact that bulk recombination currents scale at a given recombination rate /inearly with the thickness, while surface recombination should be either unaffected by thickness or even decrease in relevance. The decrease in the contribution of surface recombination would be expected since the diffusion of charge carriers to the surface becomes less efficient the further away the surface is on average $e^{28}$. Thus, if material and interface properties, like the bulk lifetime and the surface recombination velocity, stay constant as a function of absorber layer thickness, we would expect the total recombination current to scale differently in a device dominated by surface recombination than in a device dominated by bulk recombination.

From a practical perspective, we now have to decide how to compare recombination currents between devices with different thicknesses. Because we are interested in the conditions under illumination, it would be ideal to derive recombination currents from the light current-voltage curves of the solar cells under investigation. The open-circuit condition could be a suitable working point to study because the voltage at open-circuit depends entirely on the balance between generation and recombination and not like at other working points also on resistive effects. However, the thickness dependent open-circuit voltage $V_{\text {oc }}$ does not directly reveal how recombination scales with thickness because it also includes information about how photogeneration scales with thickness. Because the solar cell absorptance and therefore the total amount of charge carriers photogenerated is a strong and not always easy to predict function of absorber layer thickness, it is advisable to firstly disentangle the effects of generation and recombination. One way of doing this at open circuit is to equate the current 
densities $J_{\text {gen }}$ due to generation and $J_{\text {rec }}$ due to recombination. The current density due to recombination can be divided into an exponentially voltage dependent factor $\exp \left(q V_{\text {oc }} / k T\right)$ and a voltage independent or weakly voltage dependent factor $J_{0}$. One can then define the saturation current density $J_{0}$ via $^{29}$

$$
J_{0}=J_{\text {gen }} \exp \left(-q V_{\text {oc }} / k T\right)
$$

where $k T$ is the thermal energy, $q$ is the elementary charge, $V_{\text {oc }}$ is the open-circuit voltage and $V_{\mathrm{oc}} \gg k T / q$ is assumed. In many cases, where solar cells collect charges efficiently at short circuit, the short-circuit current density $J_{\text {sc }}$ is already a good approximation for $J_{\text {gen }}$. If this is not the case, $J_{\text {gen }}$ can be approximated by the photocurrent density $J_{\text {ph }}=J_{\text {light }}-J_{\text {dark }}$ at a sufficiently high reverse bias where collection has saturated. Here, $J_{\text {light }}$ and $J_{\text {dark }}$ are the current densities under illumination and in the dark, respectively. Note that Eq. (1) lacks the ideality factor because it assigns any voltage dependence of recombination that does not follow the ideal relation $J_{\text {rec }} \propto \exp \left(q V_{\mathrm{oc}} / k T\right)$ to a voltage dependence of $J_{0}$. It is therefore important to notice that the obtained $J_{0}$ corresponds to a specific situation in the device, namely open circuit under specific illumination conditions (i.e. the conditions used to determine $J_{\text {gen }}$ and $V_{\text {oc }}$ in Eq. (1)).

The recombination current density $J_{\text {rec }}=J_{\text {rec, }}+J_{\text {rec, B }}$ can be expressed as a sum of the contribution $J_{\mathrm{rec}, \mathrm{S}}$ from surface recombination and $J_{\mathrm{rec}, \mathrm{B}}$ from bulk recombination. The current density $J_{\text {rec, },}$ follows from the excess minority charge densities and surface recombination velocities at the electrodes via

$$
J_{\mathrm{rec}, \mathrm{S}}=q S_{\mathrm{na}} \Delta n_{\mathrm{a}}+q S_{\mathrm{pc}} \Delta p_{\mathrm{c}}
$$

where $S_{\text {na }}$ is the surface recombination velocity of electrons at the interface to the anode and $S_{\mathrm{pc}}$ is the surface recombination velocity of holes at the interface to the cathode. The surface recombination velocities $S_{\mathrm{na}}$ and $S_{\mathrm{pc}}$ have to be multiplied with the excess electron 
concentration $\Delta n_{\mathrm{a}}$ at the anode and the excess hole concentration $\Delta p_{\mathrm{c}}$ at the cathode, respectively, to obtain the surface recombination rate per area. Multiplication with the elementary charge $q$ finally yields the current density $J_{\text {rec,S }}$. The bulk recombination current density can be obtained from an integral of the bulk recombination rate $R$ over the depth of the absorber via

$$
J_{\text {rec, } \mathrm{B}}=q \int_{0}^{d} R(x) d x
$$

Here, $x$ is the spatial position in the absorber layer of thickness $d$. In addition, we can use $J_{\text {rec,S }}$ and $J_{\text {rec,B }}$ to split up the saturation current density into two parts, namely

$$
J_{0, \mathrm{~S}}=J_{\mathrm{rec}, \mathrm{S}} \exp \left(-q V_{\mathrm{oc}} / k T\right)
$$

and

$$
J_{0, \mathrm{~B}}=\mathrm{V}_{\mathrm{rec}, \mathrm{B}} \exp \left(-q V_{\mathrm{oc}} / k T\right)
$$

where $J_{0}=J_{0, \mathrm{~S}}+J_{0, \mathrm{~B}}$ is valid. While $J_{0, \mathrm{~S}}$ and $J_{0, \mathrm{~B}}$ are not accessible from experiments, it is possible to use Eqs. (2)-(5) to calculate them using numerical simulations.

Figure 1 shows the results for $J_{0}, J_{0, \mathrm{~B}}$ and $J_{0, \mathrm{~S}}$ calculated from simulated data for different conditions. The drift-diffusion simulations were performed using the electrical model of the software $\mathrm{ASA}^{30}$ together with its built-in transfer matrix code genpro1 to create a real generation profile based on the device stack glass/indium-tin oxide (ITO)/poly(3,4ethylenedioxylenethiophene):poly(4-styrenesulfonic acid) (PEDOT:PSS)/poly(3hexylthiophene):[6,6]-phenyl-C61-butyric acid methyl ester (P3HT:PC $\left.{ }_{61} \mathrm{BM}\right) / \mathrm{Ca} / \mathrm{Al}$ (see Table S1 and S2 for simulation parameters). The thicknesses of glass, ITO, PEDOT:PSS, Ca, and $\mathrm{Al}$ layers were set to $1 \mathrm{~mm}, 120 \mathrm{~nm}, 25 \mathrm{~nm}, 30 \mathrm{~nm}$, and $150 \mathrm{~nm}$ respectively. Figures 1(a) and 1(c) show the case of direct recombination only, i.e. the bulk recombination rate is given by $R=k\left(n p-n_{i}^{2}\right)$, where $k$ is the recombination coefficient, $n$ and $p$ are the electron and hole 
concentrations, respectively, and $n_{\mathrm{i}}^{2}$ is the intrinsic charge carrier concentration. Figures $1(\mathrm{~b})$ and 1(d) present the case where bulk recombination is dominated by Shockley-Read-Hall recombination and, thus, $R=\left(n p-n_{i}^{2}\right) /\left(n \tau_{\mathrm{p}}+p \tau_{\mathrm{n}}\right)$, where $\tau_{\mathrm{n}}$ and $\tau_{\mathrm{p}}$ are the electron and hole lifetimes. The results in Figures 1(a) and 1(b) correspond to the cases with a relatively lower amount of surface recombination than the cases presented in Figures $1(\mathrm{c})$ and $1(\mathrm{~d})$.

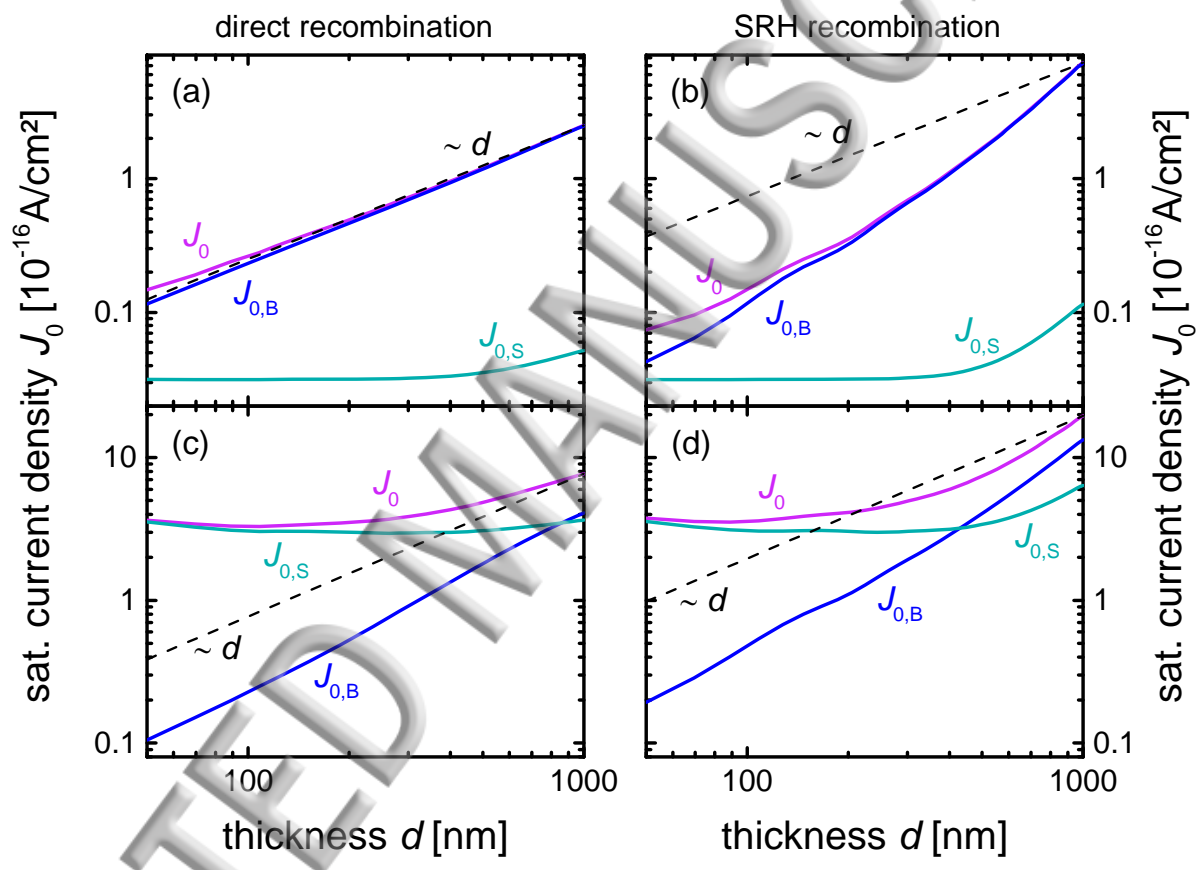

FIG. 1. Graphs showing the saturation current densities $J_{0}, J_{0, \mathrm{~B}}$ and $J_{0, \mathrm{~S}}$ calculated from simulated data for different conditions. (a) and (c) present the case of direct recombination only. (b) and (d) present the case where bulk recombination is dominated by Shockley-ReadHall recombination. The results in (a) and (b) were obtained using a relatively lower amount of surface recombination than the results in (c) and (d). In all cases the saturation current density $J_{0, \mathrm{~B}}$ due to bulk recombination increases with thickness and its slope is 1 or higher. In contrast, the saturation current density $J_{0, \mathrm{~S}}$ due to surface recombination decreases or remains constant with thickness.

In general, it can be observed that the saturation current density $J_{0, \mathrm{~B}}$ due to bulk recombination is increasing with thickness and its slope $d \ln \left(J_{0, \mathrm{~B}}\right) / d \ln (d)$ seems to be 1 or 
higher. In contrast, the saturation current density $J_{0, \mathrm{~S}}$ due to surface recombination shows a more complicated behavior. For low thicknesses $(d<100 \mathrm{~nm})$ and high surface recombination velocities $S$ (Figs. 1 (c) and $1(\mathrm{~d})$ ), $J_{0, \mathrm{~S}}$ decreases with thickness, i.e. $d \ln \left(J_{0, \mathrm{~S}}\right) / d \ln (d)<0$. This is due to the fact that the transport of charge carriers to the surface limits surface recombination $^{31}$ and this transport is more efficient when the surface is close by, i.e at low thicknesses ${ }^{28}$. In contrast, if the surface recombination velocity is small (Figs. 1(a) and 1(b)) the transport to the surface is efficient and recombination at the surface is the rate limiting step $^{31}$. In this case, $J_{0, \mathrm{~S}}$ is constant for small thicknesses.

For larger thicknesses $(d>400 \mathrm{~nm}), J_{0, \mathrm{~S}}$ increases with thickness because the optical generation is spatially inhomogeneous. At larger thicknesses, a large amount of photons will be absorbed close to the transparent front electrode that sees the light first. This will also create an inhomogeneous profile of charge carriers (even at open circuit) that will lead to increased recombination rates where the optical generation rates are higher. Because this occurs close to one of the contacts, surface recombination at this contact will become very important and lead to $d \ln \left(J_{0, \mathrm{~S}}\right) / d \ln (d)>0$.

While there is a quantitative difference between direct (Figs. 1(a) and (c)) and SRH recombination (Figs. 1(b) and 1(d)), the trends are qualitatively the same. Especially at higher thicknesses, SRH recombination allows $J_{0, \mathrm{~B}}$ to scale superlinearly with thickness, while direct recombination typically leads to a slope $d \ln \left(J_{0, \mathrm{~B}}\right) / d \ln (d)$ very close to 1 in all simulations we performed. Thus, it should be possible from studying $J_{0}$ as a function of thickness derived from experimental data to assign the dominant recombination mechanism either to the surface or the bulk (or internal interfaces in case of organic solar cells). In order to compare our simulations with experimental data, we chose organic solar cells as a model system. Organic solar cells are relatively easy to fabricate with various different device thicknesses and there is a large body of work available which can be analyzed with our method. 
Figure 2 presents the results for the saturation current density $J_{0}$ calculated using Eq. (1) on a range of experimental data from our laboratory and from literature. Figure 2(a) shows three cases where $J_{0}$ either is constant or decreases with active layer thickness. The device fabricated in our laboratory using poly[4,8-bis[(2-ethylhexyl)oxy]benzo[1,2-b:4,5b']dithiophene-2,6-diyl][3-fluoro-2-[(2-ethylhexyl) carbonyl]thieno[3,4-b]-thiophenediyl] (PTB7) as electron donor and [6,6]-phenyl-C71-butyric acid methyl ester $\left(\mathrm{PC}_{71} \mathrm{BM}\right)$ as electron acceptor and without the additive 1,8-diiodooctane (DIO) shows a saturation current density $J_{0}$ which decreases with thickness, indicating a surface limited recombination. The device made from $\mathrm{PTB} 7$ and $\mathrm{PC}_{71} \mathrm{BM}$ with a standard structure as described in Ref. ${ }^{32}$ exhibits a nearly constant value for $J_{0}$ over the entire thickness range considered. This behavior suggests a system where the transport of charge carriers to the surface is efficient and the rate limiting step is the recombination at the surface. For the device based on $\mathrm{P} 3 \mathrm{HT}$ and $\mathrm{PC}_{61} \mathrm{BM}$ blended with a $1: 1$ weight-ratio as reported in Ref. ${ }^{33}$ the slope $d \ln \left(J_{0}\right) / d \ln (d)$ is negative at low thicknesses. This trend is similar to the one presented in Figure 1(c) where the surface recombination is so high that it is limited by the transport of charge carriers to the contacts. Thus, it can be assumed that for these cells surface recombination is the dominant recombination mechanism. 


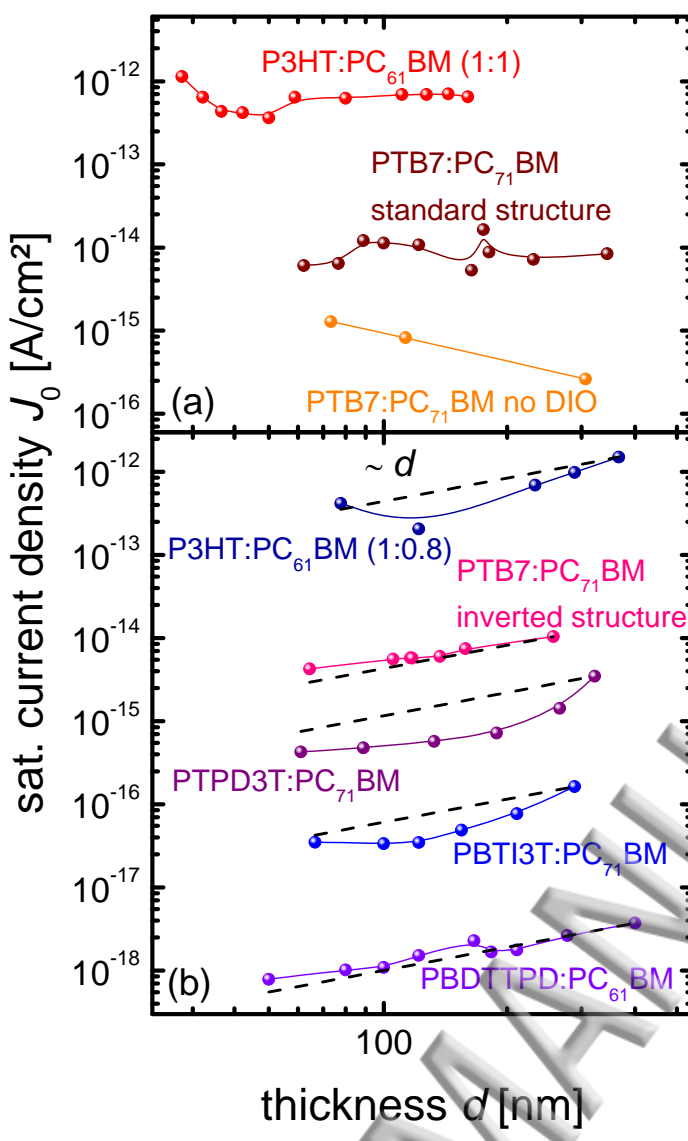

FIG. 2. Saturation current density $J_{0}$ calculated for a range of different polymer:fullerene systems. The filled circles represent the values of $J_{0}$ derived from the open-circuit voltage and the short-circuit current while the lines are just a guide to the eye. The dashed lines indicate a proportionality between $J_{0}$ and $d$, i.e. a slope of $d \ln \left(J_{0}\right) / d \ln (d)=1$. Panel (a) shows the cases where recombination is mostly limited by the surface while panel (b) presents the cases of mostly and purely bulk dominated recombination.

Figure 2(b) presents cases where recombination in the bulk plays a bigger role in determining the dominant recombination mechanism. For both self-prepared $\mathrm{PTB} 7: \mathrm{PC}_{71} \mathrm{BM}$ with DIO and an inverted structure and poly(di(2-ethylhexyloxy)benzo[1,2-b:4,5$\mathrm{b}^{\prime}$ ]dithiophene-co-octylthieno[3,4-c]pyrrole-4,6- dione) (PBDTTPD): $\mathrm{PC}_{61} \mathrm{BM}$ (fabricated as described in Ref. ${ }^{34}$ ) devices the saturation current density $J_{0}$ increases with thickness and its slope $d \ln \left(J_{0}\right) / d \ln (d)$ is approximately equal to 1 over the whole thickness range considered. This trend clearly identifies bulk recombination as the dominant recombination mechanism in 
both systems. The P3HT:PC 61 BM (1:0.8), poly[N-(2-hexyldodecyl)-2,2'-bithiophene-3,3'dicarboximide-alt-5,5-(2,5-bis(3-decylthiophen-2-yl)-thiophene)] (PBTI3T): $\mathrm{PC}_{71} \mathrm{BM}, \quad$ and poly[5-(2-hexyldodecyl)-1,3-thieno[3,4-c]pyrrole-4,6-dione-alt-5,5-(2,5-bis(3-

dodecylthiophen-2-yl)-thiophene)] (PTPD3T):PC $\mathrm{PC}_{71} \mathrm{BM}$ samples, fabricated as described in Ref. ${ }^{35},{ }^{36}$, and ${ }^{36}$, respectively, exhibit an intermediate behavior with $J_{0}$ affected by both surface and bulk recombination. In fact, the saturation current density $J_{0}$ of these three systems decreases or remains constant with $d$ for low thicknesses while it increases with $d$ for larger thicknesses. Thus, at low thicknesses the slope $d \ln \left(J_{0}\right) / d \ln (d)$ is equal to 0 or less, suggesting a bigger impact of surface recombination, and at high thicknesses $d \ln \left(J_{0}\right) / d \ln (d)$ is higher than 1, indicating that bulk recombination starts being more dominant at open circuit.

In this work we presented a method to distinguish between surface and bulk recombination in organic solar cells. This approach is based on the thickness dependence of the saturation current density which is derived from the open-circuit voltage and the photocurrent at short circuit or reverse bias. By means of numerical simulations, we showed that the bulk recombination current increases with thickness while for low thicknesses $(d<$ $400 \mathrm{~nm}$ ) the surface recombination current decreases or remains constant with thickness. This different scaling with thickness allows one to assign the dominant recombination mechanism of the solar cell under investigation either to the surface or the bulk. Finally, we checked our predictions on a range of experimental data from our laboratory and from literature showing that in the field of organic photovoltaics the whole range of cases, from mostly surface limited to purely bulk limited recombination, can be observed.

See-supplementary materials for the experimental details and the simulation parameters.

We acknowledge support from the Deutsche Forschungsgemeinschaft (DFG) (Grant No. KI$1571 / 2-1)$ 


\section{Reference list}

${ }^{1}$ B. Qi and J. Wang, J. Mater. Chem. 22, 24315 (2012).

${ }^{2}$ A. Ng, W.K. Yiu, Y. Foo, Q. Shen, A. Bejaoui, Y. Zhao, H.C. Gokkaya, A.B. Djurišić, J.A.

Zapien, W.K. Chan, and C. Surya, ACS Appl. Mater. Interfaces 6, 20676 (2014).

${ }^{3}$ D. Credgington, R. Hamilton, P. Atienzar, J. Nelson, and J.R. Durrant, Adv. Funct. Mater. 21, 2744 (2011).

${ }^{4}$ C.G. Shuttle, B. O’Regan, A.M. Ballantyne, J. Nelson, D.D.C. Bradley, and J.R. Durrant, Phys. Rev. B 78, 113201 (2008).

${ }^{5}$ K. Vandewal, K. Tvingstedt, A. Gadisa, O. Inganäs, and J. V. Manca, Nat. Mater. 8, 904 (2009).

${ }^{6}$ J.C. Blakesley and D. Neher, Phys. Rev. B 84, 075210 (2011).

${ }^{7}$ R.S. Crandall, J. Appl. Phys. 53, 3350 (1982).

${ }^{8}$ T. Kirchartz, T. Agostinelli, M. Campoy-Quiles, W. Gong, and J. Nelson, J. Phys. Chem. Lett. 3, 3470 (2012).

${ }^{9}$ S.A. Hawks, F. Deledalle, J. Yao, D.G. Rebois, G. Li, J. Nelson, Y. Yang, T. Kirchartz, and J.R. Durrant, Adv. Energy Mater. 3, 1201 (2013).

${ }^{10}$ G.F. Dibb, F.C. Jamíeson, A. Maurano, J. Nelson, and J.R. Durrant, J. Phys. Chem. Lett. 4, 803 (2013)

${ }^{11}$ C.M. Proctor, M. Kuik, and T.-Q. Nguyen, Prog. Polym. Sci. 38, 1941 (2013).

${ }^{12}$ K. Tvingstedt and C. Deibel, Adv. Energy Mater. 6, 1502230 (2016).

${ }^{13}$ M. Maiberg and R. Scheer, J. Appl. Phys. 116, 123711 (2014).

${ }^{14}$ M. Maiberg, T. Hölscher, S. Zahedi-Azad, and R. Scheer, J. Appl. Phys. 118, 105701 (2015)

${ }^{15}$ T. Kirchartz, F. Deledalle, P.S. Tuladhar, J.R. Durrant, and J. Nelson, J. Phys. Chem. Lett. 4, 2371 (2013). 
${ }^{16}$ C. Van Berkel, M.J. Powell, A.R. Franklin, and I.D. French, J. Appl. Phys. 73, 5264 (1993).

${ }^{17}$ T. Kirchartz and J. Nelson, Phys. Rev. B 86, 165201 (2012).

${ }^{18}$ T. Kirchartz, B.E. Pieters, J. Kirkpatrick, U. Rau, and J. Nelson, Phys. Rev. B 83, 115209 (2011).

${ }^{19}$ R.A. Street, A. Krakaris, and S.R. Cowan, Adv. Funct. Mater. 22, 4608 (2012).

${ }^{20}$ F. Deledalle, T. Kirchartz, M.S. Vezie, M. Campoy-Quiles, P.S. Tuladhar, J. Nelson, and J.R. Durrant, Phys. Rev. X 5, 011032 (2015).

${ }^{21}$ J. Wang, L. Xu, Y.-J. Lee, M. De Anda Villa, A. V. Malko, and J.W.P. Hsu, Nano Lett. 15, 7627 (2015).

${ }^{22}$ A. Petersen, T. Kirchartz, and T.A. Wagner, Phys. Rev. B 85, 045208 (2012).

${ }^{23}$ U. Würfel, D. Neher, A. Spies, and S. Albrecht, Nat, Commun. 6, 6951 (2015).

${ }^{24}$ S. Wheeler, F. Deledalle, N. Tokmoldin, T. Kirchartz, J. Nelson, and J.R. Durrant, Phys. Rev. Appl. 4, 024020 (2015).

${ }^{25}$ O.J. Sandberg, M. Nyman, and R. Österbacka, Phys. Rev. Appl. 1, 024003 (2014).

${ }^{26}$ B. Hoex, J. Schmidt, P. Pohl, M.C.M. van de Sanden, and W.M.M. Kessels, J. Appl. Phys. 104, 044903 (2008).

${ }^{27}$ A. Cuevas, P.A. Basore, G. Giroult-Matlakowski, and C. Dubois, J. Appl. Phys. 80, 3370 (1996).

${ }^{28}$ A.B. Sproul,J. Appl. Phys. 76, 2851 (1994).

${ }^{29}$ P.J. Rostan, U. Rau, V.X. Nguyen, T. Kirchartz, M.B. Schubert, and J.H. Werner, Sol. Energy Mater. Sol. Cells 90, 1345 (2006).

${ }^{30}$ B.E. Pieters, J. Krc, and M. Zeman, in Photovolt. Energy Conversion, Conf. Rec. 2006 IEEE 4th World Conf. (IEEE, Piscataway, NJ, 2006) (2006), pp. 1513-1516.

${ }^{31}$ O.J. Sandberg, A. Sundqvist, M. Nyman, and R. Österbacka, Phys. Rev. Appl. 5, 044005 (2016).

${ }^{32}$ H. Park, J. An, J. Song, M. Lee, H. Ahn, M. Jahnel, and C. Im, Sol. Energy Mater. Sol. 
Cells 143, 242 (2015).

${ }^{33}$ G. Li, V. Shrotriya, Y. Yao, and Y. Yang, J. Appl. Phys. 98, 043704 (2005).

34 J.A. Bartelt, Z.M. Beiley, E.T. Hoke, W.R. Mateker, J.D. Douglas, B.A. Collins, J.R. Tumbleston, K.R. Graham, A. Amassian, H. Ade, J.M.J. Fréchet, M.F. Toney, and M.D. Mcgehee, Adv. Energy Mater. 3, 364 (2013).

${ }^{35}$ D. Lee, J. Kim, S. Noh, and C. Lee, in Proc. 10th IEEE Int. Conf. Nanotechnol. Jt. Symp. with Nano Korea (2010), pp. 1175-1178.

${ }^{36}$ X. Guo, N. Zhou, S.J. Lou, J. Smith, D.B. Tice, J.W.Hennek, R.P. Ortiz, J.T.L. Navarrete, S. Li, J. Strzalka, L.X. Chen, R.P.H. Chang, A. Facchetti, and T.J. Marks, Nat. Photonics 7, 825 (2013).

${ }^{37}$ B.J. Leever, C. a. Bailey, T.J. Marks, M.C. Hersam, and M.F. Durstock, Adv. Energy Mater. 2, 120 (2012). 


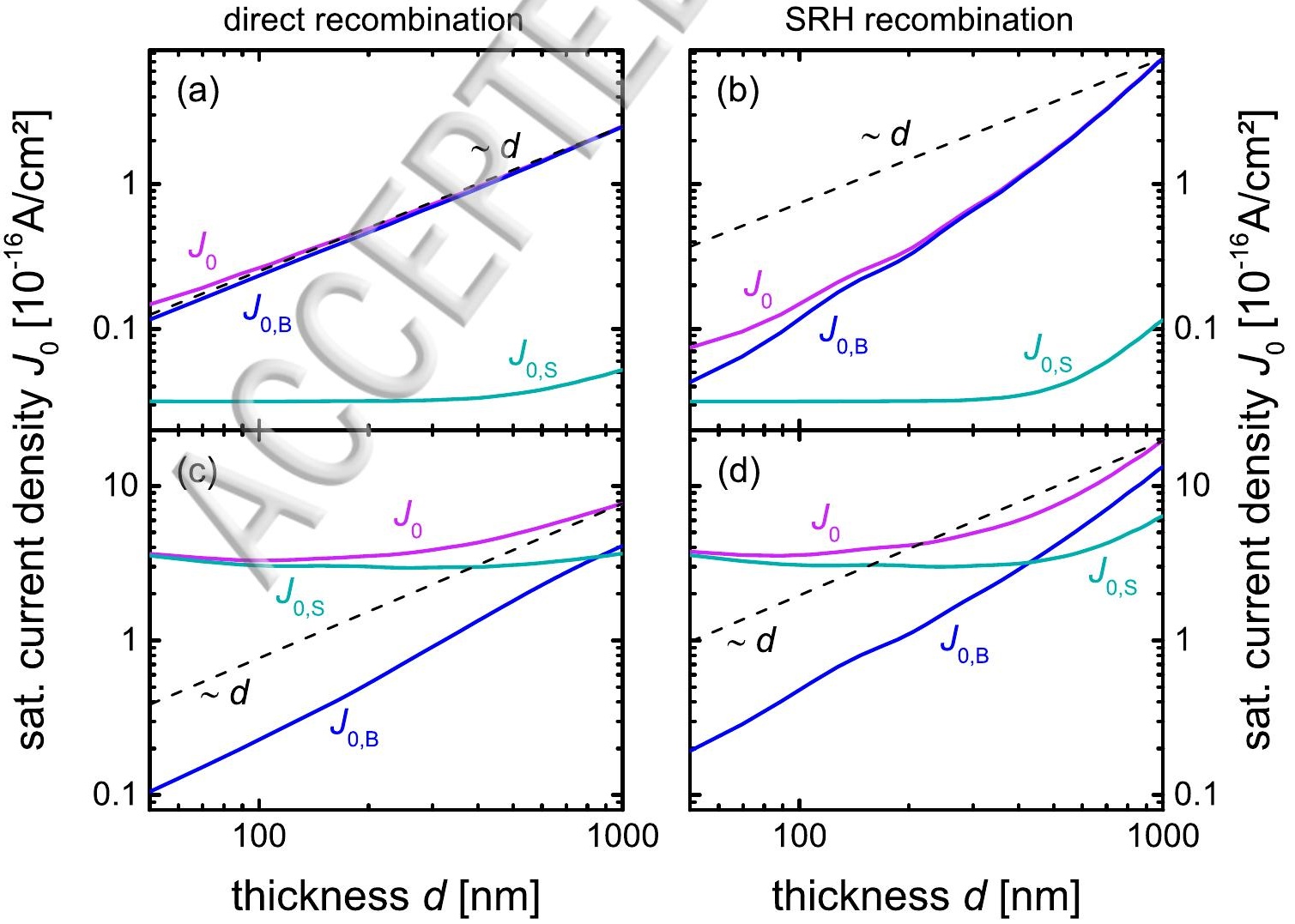




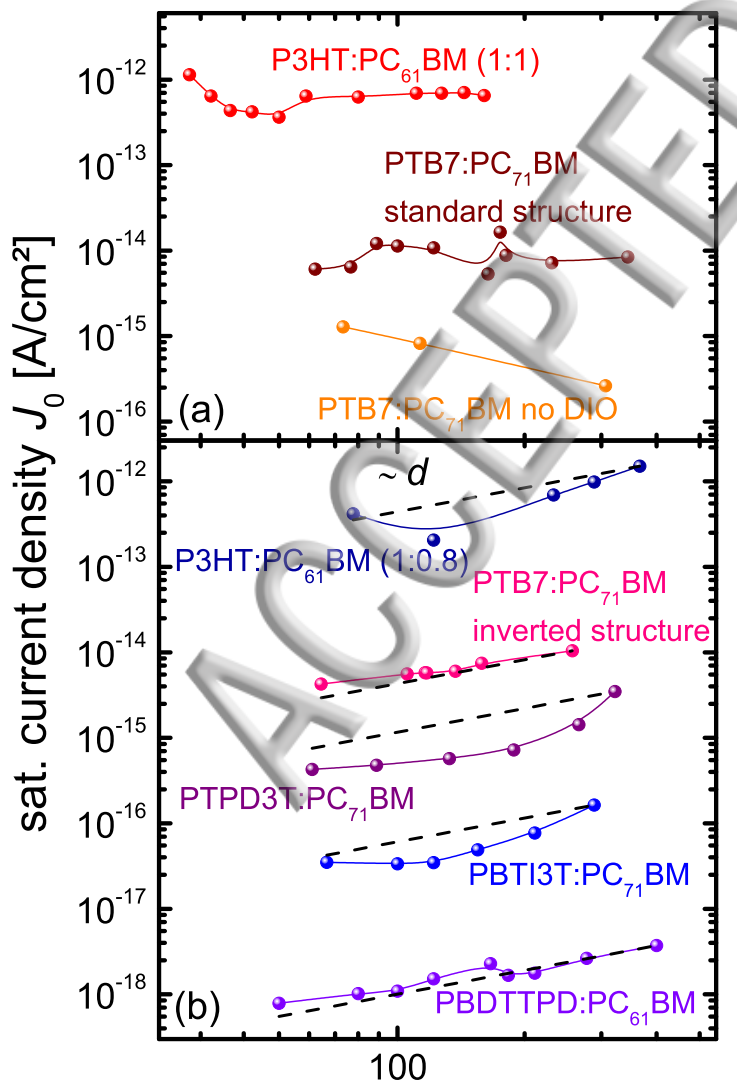

thickness $d[\mathrm{~nm}]$ 